PROCEEDINGS OF THE

AMERICAN MATHEMATICAL SOCIETY

Volume 131, Number 5, Pages 1321-1327

S 0002-9939(02)06963-0

Article electronically published on December 16, 2002

\title{
LIE ALGEBRAS AND SEPARABLE MORPHISMS IN PRO-AFFINE ALGEBRAIC GROUPS
}

\author{
NAZIH NAHLUS
}

(Communicated by Dan M. Barbasch)

\begin{abstract}
Let $K$ be an algebraically closed field of arbitrary characteristic, and let $f: G \rightarrow H$ be a surjective morphism of connected pro-affine algebraic groups over $K$. We show that if $f$ is bijective and separable, then $f$ is an isomorphism of pro-affine algebraic groups. Moreover, $f$ is separable if and only if (its differential) $f^{o}$ is surjective. Furthermore, if $f$ is separable, then $\mathcal{L}(\operatorname{Ker} f)=\operatorname{Ker} f^{o}$.
\end{abstract}

A pro-affine algebraic group $G$, over an algebraically closed field $K$, is an inverse limit of affine algebraic groups over $K$. This notion was introduced by Hochschild and Mostow in connection with the representation theory of groups [6]. For example, every complex analytic group $A$ determines a pro-affine algebraic group whose finite-dimensional rational representations are in bijective correspondence with the finite-dimensional complex analytic representations of $A$ [7] (see also [11, 13]). A similar correspondence exists for Lie algebras over $K$ in characteristic 0 [14 Remark 2, p. 139]. However, Lie algebras of pro-affine algebraic groups appear very briefly in the literature. For example, Lie algebras of finitely generated pro-unipotent groups appear in [9, p. 329] and [12, p. 171] (see also the references in [12, p. 172]).

The purpose of this paper is to extend some basic theorems (in [2 5, 8, 15]) concerning groups and their Lie algebras from the category of affine algebraic groups to the larger category of pro-affine algebraic groups. In particular, let $f: G \longrightarrow$ $H$ be a surjective morphism of connected pro-affine algebraic groups over $K$. In Theorem 1, we show that $f$ is separable if and only if its differential $f^{\circ}$ is surjective. In Theorem 4 we show that if $f$ is bijective and separable, then $f$ is an isomorphism of pro-affine algebraic groups. In Corollary 9, we show that if $f$ is separable, then $\operatorname{Ker} f^{o}=\mathcal{L}(\operatorname{Ker} f), f^{o}$ is surjective, and $f$ induces an isomorphism of pro-affine algebraic groups from $G / \operatorname{Ker} f$ onto $H$. If $\operatorname{char}(K)=0$ and $A$ and $B$ are connected algebraic subgroups of $G$, then $A \subset B$ if and only if $\mathcal{L}(A) \subset \mathcal{L}(B)$, and $A$ is normal in $G$ if and only if $\mathcal{L}(A)$ is an ideal of $\mathcal{L}(G)$ (Propositions 10,12). However, some of the results in the affine case do not extend to the pro-affine case in characteristic $p$ (see Example 15).

Received by the editors August 10, 2000.

2000 Mathematics Subject Classification. Primary 14L15, 16W30, 17B45, 20 G15.

Key words and phrases. Lie algebras of pro-affine algebraic groups, separable morphisms. 
To handle the pro-affine case as done in [6], one reduces the proof somehow to the affine case which we do in proving Theorem 8(a,b) and Propositions 1012. Roughly speaking, the reduction to the affine case depends on (i) preserving all hypotheses when we go down to the affine "components" in order to apply the theory of affine algebraic groups, and then (ii) integrating the results on the affine "components" to reach the pro-affine case. To illustrate the obstacles, let $f: G \longrightarrow H$ be a surjective morphism of pro-affine algebraic groups over $K$. By using some properties of commutative Hopf algebras, one can show that $f=\lim f_{i}$ where $\left\{f_{i}: G_{i} \longrightarrow H_{i}\right\}$ is an inverse system of surjective morphisms of affine algebraic groups. However, if $f^{o}$ is surjective (as in Theorem 1), then each $f_{i}^{o}$ may fail to be surjective. If $f$ is separable (as in Theorems 1 and 4 and Corollary 6), then each $f_{i}$ may fail to be separable (see Example 15). So the method of reduction to the affine case fails in proving Theorems 1 and 4 and (Corollary 6).

To prove Theorem 1, we shall use a certain description of the module of (Kähler) differentials in commutative Hopf algebras found in [16], while Theorem 4 is proved by a careful modification of the proof in the affine case as given by Hochschild in [5] by using certain properties of arbitrary commutative Hopf algebras over $K$ some of which were proved in [6]. We note that these properties can also be obtained as consequences of the fact that every commutative Hopf algebra over a field is faithfully flat over any of its Hopf subalgebras (see [16] as in the proof of Proposition $3)$.

We shall assume that the reader is familiar with the general properties of proaffine algebraic groups found in 6, Section 2]. We adopt the following notation and conventions: $K$ is a fixed algebraically closed field. If $G$ is a pro-affine algebraic group over $K$, then $K[G]$ is its Hopf algebra of polynomial functions [6. p. 1127], $\mathcal{L}(G)$ is the Lie algebra of $G$ [4, p. 404], and $G^{0}$ is the identity component of $G$ [4, Thm. 2.1]. If $D$ is an integral domain, $[D]$ is the field of fractions of $D$. If $A$ is an algebraic subgroup of $G$, then $K[G]^{A}$ is the $A$-fixed part of $K[G]$ under the translation given by $(x \cdot f)(y)=f(y x)$. Similarly, $[K[G]]^{A}$ is defined if $G$ is connected. As in the affine case, the elements of $G$ will be identified with the $K$-algebra homomorphisms from $K[G]$ into $K$, and the elements of $\mathcal{L}(G)$, which are the differentiations of $K[G]$, will be identified with the elements of the dual $K$-space of $I / I^{2}$ where $I$ is the augmentation ideal of $K[G]$. Moreover, if $A$ is an algebraic subgroup of $G, \mathcal{L}(A)$ will be identified with its canonical image in $\mathcal{L}(G)$.

Let $f: G \longrightarrow H$ be a surjective morphism of connected pro-affine algebraic groups, so we may identify $K[H]$ with its image in $K[G]$. Then $f$ is called separable if $[K[G]]$ is separable over $[K[H]]$.

The proof of Theorem 1 is based on the following result in [16, 11.3, p. 85].

Theorem 0. Let $A$ be a commutative Hopf algebra over a commutative ring $R$, with comultiplication $\Delta$ and augmentation ideal $I$. Let $\pi: A \longrightarrow I / I^{2}$ be the projection relative to the decomposition $A=R \oplus I$. Let $\Omega_{A}$ be the module of (Kähler) differentials of $A$. Then $\Omega_{A} \cong A \otimes I / I^{2}$, and the universal derivation $d$ is given by $d(a)=\sum a_{i} \otimes \pi\left(a_{i}^{\prime}\right)$ where $\Delta(a)=\sum a_{i} \otimes a_{i}^{\prime}$.

Theorem 1. Let $f: G \longrightarrow H$ be a surjective morphism of connected pro-affine algebraic groups over $K$. Let $f^{o}: \mathcal{L}(G) \longrightarrow \mathcal{L}(H)$ be the differential of $f$. Then $f^{o}$ is surjective if and only if $f$ is separable. 
Proof. We shall identify $K[H]$ with its canonical image in $K[G]$. Put $A=K[H]$ and $B=K[G]$. Let $I$ and $J$ be the augmentation ideals of $A$ and $B$ respectively, and let $\pi_{1}: A \longrightarrow I / I^{2}$ and $\pi_{2}: B \longrightarrow J / J^{2}$ be the projections as in Theorem 0 . Let $d_{1}: A \longrightarrow \Omega_{A}, d_{2}: B \longrightarrow \Omega_{B}, d_{1}^{\prime}:[A] \longrightarrow \Omega_{[A]}$, and $d_{2}^{\prime}:\left[B\left[\longrightarrow \Omega_{[B]}\right.\right.$ be the universal $K$-derivations of such $K$-algebras. For clarity, we shall prove our theorem by justifying the equivalence of the following highlighted statements:

(1a) $[B]$ is separable over $[A]$.

(1b) $s_{1}: \Omega_{[A]} \otimes_{[A]}[B] \longrightarrow \Omega_{[B]}$ is injective where $s_{1}$ is the canonical [B]-linear mapping given by $s_{1}\left(d_{1}^{\prime} x \otimes y\right)=y d_{2}^{\prime} x$.

(1c) $s_{2}: \Omega_{A} \otimes_{A}[B] \longrightarrow \Omega_{B} \otimes_{B}[B]$ is injective where $s_{2}$ is the canonical [B]-linear mapping given by $s_{2}\left(d_{1} x \otimes y\right)=d_{2} x \otimes y$.

(2a) $s_{3}:\left(I / I^{2} \otimes_{K} A\right) \otimes_{A}[B] \longrightarrow\left(J / J^{2} \otimes_{K} B\right) \otimes_{B}[B]$ is injective where

$$
s_{3}\left(\pi_{1}(a) \otimes a^{\prime} \otimes y\right)=\pi_{2}(a) \otimes a^{\prime} \otimes y .
$$

(2b) $s_{4}: I / I^{2} \otimes_{K}[B] \longrightarrow J / J^{2} \otimes_{K}[B]$ is injective where $s_{4}\left(\pi_{1}(a) \otimes y\right)=\pi_{2}(a) \otimes y$.

(2c) $s_{5}: I / I^{2} \longrightarrow J / J^{2}$ is injective where $s_{5}\left(\pi_{1}(a)\right)=\pi_{2}(a)$.

Now (1a) $\Leftrightarrow(1 \mathrm{~b})$ since $K$ is a perfect field [3, V, 16.4, p. 132]. (1b) $\Leftrightarrow$ (1c) since $\Omega_{[B]} \cong \Omega_{B} \otimes_{B}[B]$ and $\Omega_{[A]} \otimes_{[A]}[B] \cong\left(\Omega_{A} \otimes_{A}[A]\right) \otimes_{[A]}[B] \cong \Omega_{A} \otimes_{A}[B]$ under canonical isomorphisms. (1c) $\Leftrightarrow(2 \mathrm{a})$ by Theorem 0 since $A$ is a Hopf subalgebra of $B$. (2a) $\Leftrightarrow(2 \mathrm{~b}) \Leftrightarrow(2 \mathrm{c})$ are obvious. Hence (1a) $\Leftrightarrow(2 \mathrm{c})$. But (2c) is equivalent to the condition that the canonical map of dual $K$-spaces $\left(J / J^{2}\right)^{*} \longrightarrow\left(I / I^{2}\right)^{*}$ is surjective. Moreover, this last map can be identified with $f^{o}: \mathcal{L}(G) \longrightarrow \mathcal{L}(H)$. Hence (1a) is equivalent to the surjectivity of $f^{o}$. That is, $f$ is separable if and only if $f^{o}$ is surjective which proves Theorem 1 .

Note. If $\operatorname{char}(K)=0$ and $H$ is affine, the surjectivity of $f^{o}$ in Theorem 1 was proved in [4, p. 406]. Moreover, it was noted that the proof can be modified to the (full) pro-affine case in characteristic 0 .

Proposition 2. Let $B \subset A$ be integral domain $K$-algebras such that

(*) $A$ is finitely generated over $B$, and the $K$-algebra homomorphisms from $A$ to $K$ separate the elements of $A$.

Suppose $x$ is an element of $A$ with the property that the $K$-algebra homomorphisms from $A$ to $K$ which agree on $B$ also agree on $x$. Then $x$ is purely inseparably algebraic over $[B]$.

The proof of Proposition 2] is contained in the proof of Proposition 2.4 of [5] p. 34]. In fact, the statements of these two propositions only differ by condition $(*)$ which generalizes the original condition " $A$ is a finitely generated $K$-algebra" (by virtue of a version of Hilbert Nullstellensatz [5] p. 22]).

We shall need the following properties of commutative Hopf algebras. Let $V(A)$ denote the set of $K$-algebra homomorphisms from the $K$-algebra $A$ into $K$. If $B \subset A$ are commutative Hopf algebras over $K$ with no non-zero nilpotent elements, then $V(A)$ separates the elements of $A$ and the restriction map $V(A) \rightarrow V(B)$ is surjective [6, Thm. 2.1, p. 1131] (see also [16] Excercise 3, p. 119]).

Proposition 3. Let $B \subset A$ be integral domain Hopf algebras over $K$ such that the restriction map $V(A) \longrightarrow V(B)$ is bijective. If $[A]$ is separable over $[B]$, then $A=B$. 
Proof. Let $x$ be an element of $A$ and let $A^{\prime}$ be the Hopf subalgebra of $A$ generated by $B$ and $x$. Then $A^{\prime}$ is finitely generated over $B$ since $x$ generates an affine Hopf subalgebra of $A$ ([4, p. 400], [16, p. 24]). The above properties of commutative Hopf algebras show that $V\left(A^{\prime}\right)$ separate the elements of $A^{\prime}$. Moreover, the restriction maps $V(A) \longrightarrow V\left(A^{\prime}\right) \longrightarrow V(B)$ are both surjective. But their composition is given to be bijective. Hence $V\left(A^{\prime}\right) \longrightarrow V(B)$ is bijective. So Proposition 2 applies to see that $x$ is purely inseparably algebraic over $[B]$. Since $[A]$ is separable over $[B]$, we must have $x \in[B]$. But $[B] \cap A=B[16$. Cor. 1, p. 111]. Hence $x \in B$ which proves $A=B$.

Now we state Proposition 3 in pro-affine algebraic group terms.

Theorem 4. Let $f: G \longrightarrow H$ be a bijective separable morphism of connected proaffine algebraic groups over $K$. Then $f$ is an isomorphism of pro-affine algebraic groups.

Corollary 5. Let $f: G \longrightarrow H$ be a morphism of connected pro-affine algebraic groups. Then $f$ is an isomorphism if and only if $f$ is bijective and $f^{\circ}$ is surjective.

Proof. Combine Theorems 1 and 4 .

Corollary 6. Let $f: G \longrightarrow H$ be a surjective separable morphism of connected pro-affine algebraic groups. Then $f$ induces an isomorphism from $G / \operatorname{Ker} f$ onto $H$.

Proof. By the universal property of $G / \operatorname{Ker} f$ [6, p. 1133], $f$ factors through $G / \operatorname{Ker} f$ as $G \stackrel{\pi}{\longrightarrow} G / \operatorname{Ker} f \stackrel{f^{\prime}}{\longrightarrow} H$. Since $f$ is separable, it follows that $f^{\prime}$ is separable (because all intermediate fields in a separable field extension of a field $F$ are also separable over $F$ ). So $f^{\prime}$ is a bijective separable morphism. Hence $f^{\prime}$ is an isomorphism by Theorem 4 .

Lemma 7. Let $A$ be an algebraic subgroup of a pro-affine algebraic group $G$. Write $K[G]=\bigcup K\left[G_{i}\right]$ as a directed union where each $G_{i}$ is an affine algebraic group. Let $\rho_{i}: G \longrightarrow G_{i}$ be the canonical surjective morphism whose transpose is the inclusion map $K\left[G_{i}\right] \longrightarrow K[G]\left[6\right.$. Thm. 2.1], and let $A_{i}$ be the canonical image of $A$ in $G_{i}$ (under $\rho_{i}$ ). Then

(a) $A=\lim A_{i}$ and $\mathcal{L}(A)=\lim _{\longleftarrow} \mathcal{L}\left(A_{i}\right)$ with the evident transition morphisms in each inverse system,

(b) $\mathcal{L}(G)=\mathcal{L}\left(G^{0}\right)$, and

(c) $K\left[G_{i}\right]^{A}=K\left[G_{i}\right]^{A_{i}}$.

Proof. (a) The canonical morphism $\rho_{i}: G \longrightarrow G_{i}$ restricts to a surjective morphism $A \longrightarrow A_{i}$, so we may view $K\left[A_{i}\right]$ inside $K[A]$. Let $\alpha: K[G] \longrightarrow K[A]$ be the restriction morphism which is surjective since $A$ is an algebraic subgroup of $G$. Then $\alpha\left(K\left[G_{i}\right]\right)=K\left[A_{i}\right]$. But $K[G]=\bigcup K\left[G_{i}\right]$ as a directed union. Hence $K[A]=$ $\bigcup K\left[A_{i}\right]$ as a directed union. Consequently, $A=\lim A_{i}$ and $\mathcal{L}(A)=\lim \mathcal{L}\left(A_{i}\right)$ which proves (a).

(b) Since $\left(G^{0}\right)_{i}=\left(G_{i}\right)^{0}$ [4, Prop. 2.1], part (a) shows that $\mathcal{L}(G)=\lim \mathcal{L}\left(G_{i}\right)=$ $\lim _{\longleftarrow} \mathcal{L}\left(\left(G_{i}\right)^{0}\right)=\lim _{\longleftarrow} \mathcal{L}\left(\left(G^{0}\right)_{i}\right)=\mathcal{L}\left(G^{0}\right)$.

Finally, (c) follows immediately from the fact that $f\left(\rho_{i}(g)\right)=f(g)$ for every $f$ in $K\left[G_{i}\right]$ and $g$ in $G$ since both sides are equal to $g(f)$ when $g$ is viewed as a $K$-algebra homomorphism from $K[G]$ into $K$. This proves Lemma 7 . 
Theorem 8. Let $N$ be a normal algebraic subgroup of a connected pro-affine algebraic group $G$ over $K$. Let $\pi: G \longrightarrow G / N$ be the canonical projection. Then

(a) $\pi$ is separable and $[K[G]]^{N}=\left[K[G]^{N}\right]$,

(b) $\operatorname{Ker} \pi^{o}=\mathcal{L}(\operatorname{Ker} \pi)$, and

(c) $\pi^{o}$ is surjective and $\mathcal{L}(G / N) \cong \mathcal{L}(G) / \mathcal{L}(N)$.

Proof. We shall reduce the proofs of (a) and (b) to the affine case. Let $K[G]=$ $\bigcup K\left[G_{i}\right], \rho_{i}: G \longrightarrow G_{i}$, and $N_{i}$ be the canonical image of $N$ in $G_{i}$ as in Lemma 7 . We shall make the identifications $K\left[G_{i} / N_{i}\right]=K\left[G_{i}\right]^{N_{i}}$ and $K[G / N]=K[G]^{N}$ as in [6. p. 1133].

(a) Since $N_{i}$ is a normal algebraic subgroup of the connected affine algebraic group $G_{i}, G_{i} / N_{i}$ is affine, so $\left[K\left[G_{i}\right]\right]^{N_{i}}=\left[K\left[G_{i}\right]^{N_{i}}\right]$ by [1, Thm. 4, p. 136]. We also have $K[G]=\bigcup K\left[G_{i}\right]$ as a directed union. Hence by Lemma $7(\mathrm{c}),[K[G]]^{N}=$ $\bigcup\left[K\left[G_{i}\right]\right]^{N_{i}}=\bigcup\left[K\left[G_{i}\right]^{N_{i}}\right]=\left[K[G]^{N}\right]$. Thus $[K[G]]^{N}=\left[K[G]^{N}\right]$. But $[K[G]]$ is separable over $[K[G]]^{N}$ by $[3$, p. 121] and [5, p. 33]. Hence $[K[G]]$ is separable over $[K[G / N]]$ so the canonical projection $\pi: G \longrightarrow G / N$ is separable.

(b) Let $\pi_{i}: G_{i} \longrightarrow G_{i} / N_{i}$ be the canonical projection. Then $\left(\pi_{i} \circ \rho_{i}\right)\left(N_{i}\right)=\{1\}$, so the universal property of $G / N$ in [6, p. 1133] shows that $\pi_{i} \circ \rho_{i}$ factors through $G / N$ as shown in the following commutative diagram:

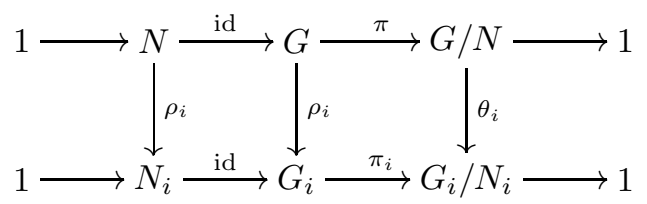

From Lemma 7(a) we know that $N=\varliminf^{\lim } N_{i}$ and $\mathcal{L}(N)=\lim \mathcal{L}\left(N_{i}\right)$ with the evident transition morphisms in each inverse system. Similarly, $G=\lim G_{i}$ and $\mathcal{L}(G)=$ $\lim \mathcal{L}\left(G_{i}\right)$. We also have $K[G]^{N}=\bigcup K\left[G_{i}\right]^{N_{i}}$ by Lemma $7(\mathrm{c})$. Thus $K[G / N]=$ $\bigcup K\left[G_{i} / N_{i}\right]$ as a directed union. Hence $G / N=\lim G_{i} / N_{i}$ and $\mathcal{L}(G / N)=$ $\lim _{\mathcal{L}} \mathcal{L}\left(G_{i} / N_{i}\right)$ with the evident transition morphisms in each inverse system. Consequently the sequence

$$
0 \longrightarrow \mathcal{L}(N) \stackrel{(\mathrm{id})}{\longrightarrow} \mathcal{L}(G) \stackrel{\pi^{o}}{\longrightarrow} \mathcal{L}(G / N) \longrightarrow 0
$$

is the inverse limit sequence of the sequence of inverse systems

$$
0 \longrightarrow\left(\mathcal{L}\left(N_{i}\right)\right) \stackrel{(\mathrm{id})}{\longrightarrow}\left(\mathcal{L}\left(G_{i}\right)\right) \stackrel{\left(\pi_{i}^{o}\right)}{\longrightarrow}\left(\mathcal{L}\left(G_{i} / N_{i}\right)\right) \longrightarrow 0 .
$$

But this is an exact sequence by the theory of affine algebraic groups ( $[2,5.6], 8$ 11.5 , p. 82]). Moreover, it is well known that $\lim$ is a left exact functor. Hence $\operatorname{Ker} \pi^{o}=\mathcal{L}(N)=\mathcal{L}(\operatorname{Ker} \pi)$ which proves (b). Finally, since $\pi: G \longrightarrow G / N$ is separable by part (a), Theorem 1 shows that $\pi^{o}$ is surjective. Consequently, $\mathcal{L}(G / N) \cong \mathcal{L}(G) / \mathcal{L}(N)$. This completes the proof of Theorem 8 .

Corollary 9. Let $f: G \longrightarrow H$ be a separable surjective morphism of connected proaffine algebraic groups. Then $\operatorname{Ker} f^{o}=\mathcal{L}(\operatorname{Ker} f), f^{o}$ is surjective, and $f$ induces an isomorphism of pro-affine algebraic groups from $G / \operatorname{Ker} f$ onto $H$.

Proof. Combine Theorems 1 and 8 with Corollary 6 . 
Proposition 10. Let $A$ be a connected algebraic subgroup of a connected pro-affine algebraic group $G$ over $K$. If $A$ is normal in $G$, then $\mathcal{L}(A)$ is an ideal of $\mathcal{L}(G)$. Moreover, the converse is true if char $(K)=0$.

Proof. The proof is obtained by reduction to the affine case. Let $\rho_{i}: G \longrightarrow G_{i}$ and $A_{i}=\rho_{i}(A)$ as in Lemma 7. Then $A=\lim A_{i}, \mathcal{L}(A)=\lim \mathcal{L}\left(A_{i}\right), G=\lim G_{i}$ and $\mathcal{L}(G)=\lim \mathcal{L}\left(G_{i}\right)$ by Lemma $7(\mathrm{a})$. Now suppose $A$ is normal in $G$. Then each $A_{i}$ is normal in $G_{i}$ since each $\rho_{i}$ is surjective. So each $\mathcal{L}\left(A_{i}\right)$ is an ideal of $\mathcal{L}\left(G_{i}\right)$. Consequently, $\lim \mathcal{L}\left(A_{i}\right)$ is an ideal of $\lim \mathcal{L}\left(G_{i}\right)$. Hence $\mathcal{L}(A)$ is an ideal of $\mathcal{L}(G)$. Conversely, suppose $\operatorname{char}(K)=0$ and $\overleftarrow{L}(A)$ is an ideal of $\mathcal{L}(G)$. Then $\rho_{i}^{o}(\mathcal{L}(A))$ is an ideal of $\rho_{i}^{o}(\mathcal{L}(G))$ for each $i$. By Theorem $1, \rho_{i}^{o}$ maps $\mathcal{L}(A)$ onto $\mathcal{L}\left(A_{i}\right)$ and $\mathcal{L}(G)$ onto $\mathcal{L}\left(G_{i}\right)$. Hence each $\mathcal{L}\left(A_{i}\right)$ is an ideal of $\mathcal{L}\left(G_{i}\right)$, so each $A_{i}$ is normal in $G_{i}$. Hence $\lim A_{i}$ is normal in $\lim G_{i}$. Consequently, $A$ is normal in $G$ which proves Proposition 10 .

Similarly, by reduction to the affine case as in the proof of Proposition 10, we obtain the following.

Proposition 11. Let $G$ be a connected pro-affine algebraic group over $K$. If $G$ is abelian, then $\mathcal{L}(G)$ is abelian. Moreover, the converse is true if $\operatorname{char}(K)=0$.

Proposition 12. Let $A$ and $B$ be connected algebraic subgroups of a connected pro-affine algebraic group $G$ over $K$. If $\operatorname{char}(K)=0$, then $A \subset B$ if and only if $\mathcal{L}(A) \subset \mathcal{L}(B)$.

Corollary 13. Let $f: G \longrightarrow H$ be a morphism of connected pro-affine algebraic groups over $K$ such that $f^{o}$ is surjective. If $\operatorname{char}(K)=0$, then $f$ is surjective.

Now we recall the following definition. A morphism $f: G \longrightarrow H$ of connected pro-affine algebraic groups over $K$ is called a covering if $f$ is surjective, Ker $f$ is profinite, and the bijective morphism $G / \operatorname{Ker} f \longrightarrow H$ induced by $f$ is an isomorphism of pro-affine algebraic groups [4, p. 402].

Corollary 14. Let $f: G \longrightarrow H$ be a morphism of connected pro-affine algebraic groups over $K$. If $f$ is a covering, then $f^{o}$ is bijective. Moreover, the converse is true if $\operatorname{char}(K)=0$.

Proof. If $f$ is a covering, then $f^{o}$ is bijective by Theorem 8 and Lemma 7(b). Conversely, if $f^{o}$ is bijective and $\operatorname{char}(K)=0$, then $f$ is surjective by Corollary 13. Moreover, Ker $f$ is pro-finite by Corollary 9, Proposition 12 and Lemma 7(b). Finally, the bijective separable morphism $G / \operatorname{Ker} f \longrightarrow H$ is an isomorphism by Theorem 4

Example 15. Assume $K$ to be of prime characteristic. As in Example 3.9 of 10 p. 49], consider the integral domain Hopf algebra $A=\lim _{\longrightarrow} A_{i}$ and $(i \in \mathbf{N})$ where $A_{i}$ is the Hopf algebra $K[x]$ of the additive group $K$, and $\vec{A}_{i} \longrightarrow A_{i+1}$ by $x \longrightarrow x^{p}$. Then $I(A)=I(A)^{2}$ where $I(A)$ is the augmentation ideal of $A$. Hence the pro-affine algebraic group $G$ whose $K[G]=A$ has a zero Lie algebra although $G$ is nontrivial connected.

For another description of $G$, let $G=\lim G_{i}$ where $G_{i}$ is the additive group $K$ and $G_{i+1} \longrightarrow G_{i}$ is given by $x \longrightarrow x^{p}$. Again, $\mathcal{L}(G)=\{0\}$ since $\mathcal{L}(G)=\lim \mathcal{L}\left(G_{i}\right)$ where each transition map is the zero map. If $f:\{1\} \longrightarrow G$ is the trivial morphism, then $f^{o}$ is surjective although $f$ is not surjective. 
Note that such types of failure never occur in the affine case for reasons of dimension.

\section{ACKNOWLEDGEMENTS}

It is a pleasure to express my thanks to Professors G. Hochschild and A. R. Magid for introducing me to some of their work and for many stimulating discussions on various occasions. In the special case of characteristic 0, the result in Corollary 9

was obtained during the author's sabbatical leave at the University of California Berkeley during 1998-1999.

\section{REFERENCES}

1. A. Bialynicki-Birula, G.Hochschild and G.D. Mostow, Extensions of representations of algebraic groups, Amer. J. Math. 85 (1963), 131-144. MR 27:5871.

2. A. Borel, Linear algebraic groups, second edition, GTM 126, Springer-Verlag, 1991. MR 92d:20001

3. N. Bourbaki, Algebra II, Chapters 4-7, Springer-Verlag, 1990. MR 91h:00003

4. G. Hochschild, Coverings of pro-affine algebraic groups, Pacific J. Math. 35 (1970), 399-415. MR 43:4830

5. — Basic theory of algebraic groups and Lie algebras, GTM 75, Springer Verlag, 1981. MR 82i:20002

6. G. Hochschild and G.D. Mostow, Pro-affine algebraic groups, Amer. J. Math. 91 (1969), 1127-1140. MR 41:350

7. Complex analytic groups and Hopf algebras, Amer. J. Math. 91 (1969), 1141-1151. MR 40:5782

8. J. E. Humphreys, Linear algebraic groups, GTM 21, Springer-Verlag, 1975. MR 53:633

9. A. Lubotzky and A. R. Magid, Free prounipotent groups, J. Algebra 80 (1983), 323-349. MR 85b:14062

10. A. R. Magid, The universal group cover of a pro-affine algebraic group, Duke Math. J. 42 (1975), 43-49. MR 51:8275

11. __ Module categories of analytic groups, Cambridge Univ. Press, 1982. MR 84j:22008

12. A. R. Magid, Prounipotent prolongation of algebraic groups, Contemp. Math. J. 224 (1999), 169-187. MR 99k:20097

13. N. Nahlus, Representative functions on complex analytic groups, Amer. J. Math. 116 (1994), 621-636. MR 95g:22009

14. $ـ$ Basic groups of Lie algebras and Hopf algebras, Pacific J. Math. 180 (1997), 135-151. MR 99g: 17053

15. T. A. Springer, Linear algebraic groups, second edition, Birkhäuser, 1998. MR 99h:20075

16. W. Waterhouse, Introduction to affine group schemes, GTM 66, Springer-Verlag, 1979. MR 82e: 14003

Department of Mathematics, American University of Beirut, Beirut, Lebanon

E-mail address: nahlus@aub.edu.lb 